\title{
BIOMARCADORES SALIVARES DO CARCINOMA DE CÉLULAS ESCAMOSAS
}

\author{
Salivary biomarkers of squamous cell carcinoma
}

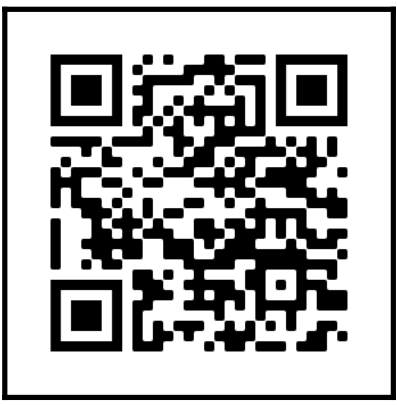

\section{Autores:}

Helba Pires Miranda

Cirurgiã Dentista - Universidade Salgado de Oliveira

\section{Bruno Pires Miranda}

Mestre em Odontologia - UFF Especialista em implantodontia - UFF

\section{Monica Lage da Rocha}

Doutora em Patologia-Buco- Dental - UFF

Mestre em Patologia - UFF

\section{William Napolitano Correa}

Mestre em Patologia-Buco-Dental - UFF

Especialista em Estomatologia - UFRJ

Prof. Dr. Marcos da Veiga Kalil

Professor Adjunto do Departamento de Odontoclínica - UFF

Doutor em endodontia - UERJ Especialista em implantodontia, Especialista em periodontia, Especialista em radiologia odontológica e Especialista em gestão da educação - UFF Mestre em clínica odontológica - UFF

\section{Endereço para correspondência:}

Bruno Pires Miranda

Rua Desembargador Lima Castro, 19 - sobrado - Fonseca, Niterói - RJ CEP: 24.120-350 Telefone: (21) 99826-0202

E-mail: brunopiresmiranda7@gmail.com 
Palavras-chaves: Carcinoma de células escamosas. Boca. Biomarcadores. Saliva. Saúde bucal.

Keywords: Carcinoma, Squamous Cell. Mouth. Biomarkers. Saliva. Oral health.

\section{INTRODUÇÃO}

O carcinoma de células escamosas (OSCC) é uma neoplasia maligna que atinge a cavidade oral, lábios e orofaringe com uma das maiores taxa de mortalidade em todo o mundo, quando em comparação com outros carcinomas, o que o torna um problema de saúde pública (MORO, 2018). Devido à sua grande prevalência, os pesquisadores buscam por mecanismos para aprimorar o diagnóstico da doença em sua fase inicial, no intuito de possibilitar melhor qualidade de vida e sobrevida a esses pacientes (CHENG et al., 2014).

Tem-se desenvolvido estudos através da expressão de proteínas, envolvendo o mecanismo biomolecular da carcinogênese oral na busca de identificação de biomarcadores que tenham potencial preditivo e um bom prognóstico para OSCC (CARVALHO; OLIVEIRA, 2015; LOUSADA-FERNANDEZ et at., 2018).

Em uma revisão sobre o genoma salivar do câncer oral, este destacou-se justamente pelo fato de suas proteínas estarem localmente expressas (SHAH et at., 2011), tornando seus biomarcadores salivares de fácil e rápida coleta, já que a proliferação desordenada de células malignas deixa derivados de DNA, RNA, vesiculas (exossomos) e marcadores proteicos nos fluidos creviculares, podendo demostrar de forma preventiva o câncer. Com isso, esses biomarcadores podem ser identificados na fase inicial da doença, ao contrário do exame clínico, já que as manifestações clínicas são tardias, o que gera um prognóstico ruim (LOUSADA-FERNANDEZ et al., 2018).

Diante desse quadro, o objetivo deste trabalho foi fazer uma revisão de literatura acerca do papel dos biomarcadores tumorais e discutir sobre a atual situação do câncer bucal, justificando o uso de biomarcadores salivares e entendendo seu papel no diagnóstico precoce.

Para isso foi feita uma busca detalhada nas bases de dados Pubmed-Medline, Biblioteca Virtual em Saúde, Lilacs e Scielo, para identificar os mais recentes 
biomarcadores citados nas literaturas ultilizando as palavras-chaves: carcinoma, biomarcadores, saliva.

Também foram incluídos dados coletados da Organização Mundial da Saúde e do Instituto Nacional do Câncer.

\section{REVISÃO DE LITERATURA}

\section{Situação Atual do OSCC}

O OSCC, também conhecido como carcinoma epidermoide, é uma neoplasia maligna de origem do epitélio de revestimento com modificação e proliferação desordenada de células, e corresponde $94 \%$ de todas as malignidades da cavidade oral (CHI, 2009).

A carcinogênese oral é um processo de múltiplos estágios que apresenta uma alta tendência de recorrer localmente ou disseminar-se por metástase, surgindo em locais distantes do de sua origem, mesmo após as terapias locais. Estas muitas vezes precedem de forma concomitante e agressiva onde as células tumorais malignas se proliferam e exercem perda de controle do ciclo celular (SINEVICI; O'SULLIVAN, 2016).

O OSCC é uma das doenças com maiores taxas de mortalidade e incidência no mundo. Em 2018, dados coletados pelo GLOBOCAN 2018 (um banco de dados online que fornece estimativas de incidência e mortalidade em 185 países para 36 tipos de câncer) foram compilados e divulgados pela Agência Internacional de Pesquisa sobre o Câncer (IARC), no intuito de prever a incidência global de câncer oral em 2018. Estimou-se 18,1 milhões de novos casos de câncer (17 milhões excluindo câncer de pele não melanoma) e 9,6 milhões de mortes por câncer (9,5 milhões excluindo câncer de pele não melanoma) em todo o mundo em 2018 (FERLAY et al., 2019).

Até 2040, a carga global deverá crescer para 27,5 milhões de novos casos de câncer e 16,3 milhões de mortes, simplesmente devido ao crescimento e envelhecimento da população. A carga futura provavelmente será ainda maior devido ao aumento da prevalência de fatores que aumentam o risco, como tabagismo, dieta não saudável, inatividade física e menos partos, em países em transição econômica (AMERICAN CANCER SOCIETY, 2019). 
No Brasil, apenas em 2015, ocorreram 5.898 óbitos decorrentes de OSCC, e estimam-se 14.700 casos novos de câncer da cavidade oral em cada ano do biênio 2018-2019, ocupando a 5a posição mais frequente entre todos os cânceres entre os homens e a 12a entre as mulheres. Devido a esse aumento, é considerado um problema de saúde pública (INCA, 2018).

Segundo a Organização Mundial de Saúde (OMS, 2020) apesar de muitos progressos na prevenção, tratamento e nos cuidados paliativos da doença, as taxas de incidência e mortalidade continuam altas, devido ao acesso a esses avanços tecnológicos serem desiguais em muitos países, fazendo com que o câncer continue sendo diagnosticado muito tarde. Além disso, o tratamento é caro ou inacessível e serviços paliativos não estão disponíveis para todos.

O diagnóstico tardio da doença é a grande razão de baixas taxas de sobrevida, sendo de cerca de $62 \%$ em média, cinco anos após o diagnóstico (CHENG et al., 2014). Apesar da gravidade patológica a descoberta precoce da doença pode amenizar o sofrimento do paciente e possibilitar um tratamento menos invasivo com melhor prognóstico (SAXENA et al., 2017).

\section{Saliva como Meio Diagnóstico}

A saliva é um fluido biológico complexo encontrado na cavidade oral, constituído por $99 \%$ de base de água, $0,3 \%$ de proteínas e $0,2 \%$ de substâncias inorgânicas (sódio, potássio, cálcio, magnésio, cloretos e carbonatos) e orgânicas (amilases, peroxidase, lipase, mucinas, lisozimas), com pH em torno de 6,7, ou seja, normalmente ácido, sendo produzida pelas três principais glândulas salivares (parótida, submandibular e sublingual) e glândulas menores (labial, lingual, palatino e tecidos bucal). Estima- se que um indivíduo saudável secrete, diariamente, em torno de 1,5 litro de saliva (KACZOR-URBANOWICZ et at., deglutição, degustação, digestão e lubrificação tecidual. Também mantem a integridade dos elementos dentários, através da capacidade tamponamento salivar do $\mathrm{pH}$ e remineralização. Ainda é composta por citocinas, enzimas, hormônios, anticorpos, antibacterianos e antivirais, que são transportados pelo sangue até a ultrafiltração das glândulas salivares, usando vias paracelulares ou através do sulco gengival (KHURSHID et al., 2016). Portanto, a saliva contém diferentes tipos de moléculas, que são facilmente coletadas, podendo ser utilizada como meio de diagnóstico (KHAN et al., 2018). 
A biópsia líquida, onde se coleta os fluidos corporais como exame diagnóstico, é uma técnica ainda em desenvolvimento, mas que começou a ser aplicada em alguns hospitais em meados de 2016, despertando interesse em profissionais da saúde e pacientes. Principalmente quando se trata da vigilância de um carcinoma (CHENG et al., 2019).

A biopsia líquida visa fornecer uma alternativa através de biomarcadores, que podem estar presentes nos fluidos corporais como sangue, saliva e urina, os quais dizem muito a respeito da saúde sistêmica e geral do paciente. Todavia, um maior impulso dos pesquisadores no desenvolvimento de estudos sobre biomarcadores salivares se justifica pela vantagem de ser um método não invasivo e com grande perspectiva de diagnosticar precocemente algumas patologias graves, como o OSCC (BANO; DAVID; INDIRA, 2015; RADHIKA et al., 2016).

Biomarcador pode ser definido como sendo uma molécula biológica possível de ser encontrada no sangue ou em outros fluídos corporais, nos produtos decorrentes do metabolismo e nos tecidos. São moléculas capazes de distinguir processos compatíveis com saúde ou doença (GUPTA.2015).

Os biomarcadores foram recentemente definidos como uma estrutura ou substância apta a ser quantificada e avaliada para demostrar, no organismo, seus produtos derivados, com a capacidade de identificar uma determinada doença, e monitorar o estado de saúde do indivíduo e reconhecer a resposta do organismo a uma intervenção terapêutica (BANO et al., 2015).

No entanto, a dinâmica do gradiente da abundância de proteínas entre tecido (tumor invasivo e tumor interno) e saliva, a abundância de proteínas na saliva e sua associação com o prognóstico não estão necessariamente associadas a proximidade do epitélio oral alterado (CARNIELLI et al, 2018).

De qualque forma, a saliva como meio diagnóstico é um método não invasivo que não confere nenhum desconforto ao paciente, podendo ser realizado periodicamente, sendo de bom aceite, principalmente pelos pacientes geriátricos e pediátricos, que se apresentam como os menos cooperativos (WANG et at., 2016), pois como não há necessidade de usar agulhas para a coleta de amostra, é mais confortável para o paciente, uma vez que reduz os níveis de ansiendade (KACZOR- URBANOWICZ et at., 2017).

É de fácil de armazenamento porque, ao contrário do sangue, não coagula. É mais seguro para o técnico/profissional, pois possui baixo risco de 
contaminação (WANG et at., 2016), já que o risco de lesão percutânea e autocontenção é evitado, por não utilizar agulhas (KACZOR- URBANOWICZ et at., 2017).

Portanto, há muitas vantagens de usar a saliva como diagnóstico, pois sua coleta é rápida, fácil, barata e não invasiva, sendo fácil de armazenar e de transportar, não coagula como o sangue e pode refletir o estado atual de saúde de um indivíduo (KACZOR-URBANOWICZ et at., 2017).

Assim, a saliva serve como meio de diagnóstico para diversos tipos de câncer, não apenas o OSCC, entre eles o câncer de esôfago, estômago, intestino grosso, ovário, endométrio, pâncreas, gastrointestinais, pulmão e mama, porém com anticorpos tumorais diferentes (KACZOR-URBANOWICZ et at., 2017)

Porém, há alguns problemas e desafios que precisam ser solucionados para total estabelecimento deste método. No uso clínico incluem: a falta de padronização para coleta, processamento e armazenamento das amostras de saliva; e ampla variabilidade de níveis de potenciais biomarcadores nos indivíduos não- cancerígenos que têm uma doença inflamatória oral crônica (AZIZ et al., 2015).

\section{DISCUSSÃO}

O OSCC é uma das neoplasias malignas em que a biopsia líquida fornece uma vantagem a mais, devido à sua estreita relação com o câncer bucal e com proteínas expressas localmente, que estão disponíveis na saliva, como a metaloproteinase de matriz (MMP) e interleucina (IL), que fazem da saliva uma poderosa ferramenta de investigação de escolha como potenciais biomarcadores para o câncer bucal (KHAN et at., 2018).

Existem duas formas de estudar o papel da biomarcadores de OSCC: pelo grau de displasia epitelial ou pelo padrão distributivo dos biomarcadores (RADHIKA et at., 2016). A classificação se baseia, em um primeiro momento, em mudanças no DNA celular que conduzem a transcrições alteradas de RNA e, consequentemente, levam a alterações na quantidade das proteínas que são expressas no meio intracelular, extracelular e na superfície da célula (BANO et al., 2015.; RADHIKA et al., 2016). A Tabela 1 apresenta as formas que podem ser estudados os biomarcadores: baseados em biomoléculas ou no estado de doença. 
Tabela 1: Formas de serem estudados os biomarcadores

\begin{tabular}{|c|c|}
\hline Baseado em biomoléculas & Baseado no estado de doença \\
\hline Biomarcadores de DNA & Biomarcadores de previsão \\
\hline Biomarcadores de RNA & Biomarcadores de deteç̃ão \\
\hline Biomarcadores de proteínas & Biomarcadores de diagnóstico \\
\hline Biomarcadores de Glyco & Biomarcadores prognósticos \\
\hline
\end{tabular}

Fonte: Elaborada pelos autores.

Atuamente existem mais de 100 biomarcadores para o OSCC referênciados na literatura. Foram selecionados alguns dos principais, segundo RADHIKA et at. (2016), como visto na tabela 2.

Tabela 2: Biomarcadores para o OSCC.

\begin{tabular}{|c|c|c|}
\hline Marcadores DNA & Marcadores RNA & Marcadores proteicos \\
\hline $\begin{array}{l}\text { Perda alélica } \text { no } \\
\text { cromossomo. Mutações do } \\
\text { DNA mitocondrial. } \\
\text { Amplificação do gene da } \\
\text { ciclina D1. } \\
\text { Aumento do marcador Ki67. } \\
\text { Presença de genomas de } \\
\text { HPV. }\end{array}$ & $\begin{array}{l}\text { Presença de IL-8, IL-1B } \\
\text { Proteina de ligação ao cálcio } \\
\text { S100 P (S100 P) } \\
\text { Familia de histonas H3 3A } \\
\text { (H3F3A) } \\
\text { MiR-125, miR-200, miR-31 }\end{array}$ & $\begin{array}{l}\text { Aumento na IL-6, IL 8, CD-44 } \\
\text { Inibidores da apoptose (IAP) } \\
\text { Antígeno associado ao SCC } \\
\text { (SCC-Ag) } \\
\text { Defensina-1 } \\
\text { Antígeno Carcio embrionário - } \\
\text { Marcador de tumor sérico } \\
\text { (CEA125) } \\
\text { (cyfra-21) } \\
\text { MMPs - }(2,9) \text { - a amilase, fator } \\
\text { de necrose tumoral - TNF-a, } \\
\text { Catalase, transferrina, a } \\
\text { amilase. }\end{array}$ \\
\hline
\end{tabular}

Fonte: Adaptado e modificado de Radhika et at., 2016.

Os estudos já efetuados, em sua maioria, tiveram base em comparação de indivíduos diagnosticados com lesões pré-malignas e malignas e indivíduos saudáveis, sendo relevante o nível das concentrações dos biomarcadores (BELLAIRS; HASINA; AGRAWAL, 2017).

Por exemplo, as defensinas são peptideos catiónicos, produzidas pelos tecidos orais e glândulas salivares sendo atribuidas na saliva e no fluido crevicular, podendo ser dectatadas mesmo em seu estágios iniciais. São antimicrobianas e citotóxicas (RADHIKA.et at., 2016). Estas proteínas são encontradas em grânulos azurofílicos de leucócitos polimorfonucleares. Estas mostraram ter uma forte associação com maior risco de desenvolvimento de OSCC (GUPTA, 2017).

A Ciclina D1, proteína que controla a proliferação e diferenciação celular, fator de transformação do crescimento (TGF- P), é considerado o ponto de partida das alterações comuns presentes no DNA, displasias e células cancerígenas (BELLAIRS; HASINA; AGRAWAL, 2017). 
Entre todos os possíveis fatores etiológicos do OSCC, o papilomavírus humano (HPV) desempenha um papel significativo. Assim, a presença de HPV, bem como do vírus Epstein- Barr (EBV), foram identificadas como possíveis marcadores de DNA na detecção de OSCC e progressão do tumor (GUPTA, 2017).

Outros biomarcadores salivares que se encontram significativamente alterados em pacientes com OSCC são os inibidores de apoptose, SCC-Ag, RNS, IGF, lactato desidrogenase e imunoglobulina $\mathrm{G}$, antígeno específico do polipeptídeo tecidual (PPS), antígeno carcinoembrionário e carcinoantígeno (CA19-9, 128). (GUPTA, 2017). Segundo Gupta (2017) e Saxena et at. (2017), o gene p 53 é um achado comum em 50 a $70 \%$ das células cancerígenas, e é o promotor de hipermetilação.

O número de marcadores proteicos, expressos na saliva, que sofrem alterações durante o desenvolvimento de lesões pré-malignas e malignas de câncer oral é muito elevado (KHAN et at., 2016). Sabe-se que as Citoquinas, de uma forma geral, nas condições inflamatórias induzem o processo de cicatrização e reparação tecidual. Quando se está perante células cancerígenas, isso não acontece, pois as citoquinas diminuem a resposta imune, inibindo a reparação tecidual e influenciando de uma forma negativa os mecanismos de regulação, proliferação e diferenciação celular material promovendo o processo de angiogênese na progressão da malignização (BANO et al., 2015). Esta proteína está associada ao processo de carcinogênese com a proposta de marcadores prognósticos em pacientes portadores de lesões orais malignas e pré-malignas, que apresentam elevados níveis destas proteínas no sangue (SAHIBZADA et al., 2017).

Os exossomos salivares são pequenas vesículas extracelulares com 30-100nm de diâmetro, consideradas mediadores/sinalizadores intercelulares, agindo como biomarcadores do câncer (CHENG; NONAKA; WONG, 2019). Segundo Kaczor-Urbanowicz et at. (2017), esses exossomos salivares (Evs) podem ser o caminho por onde a informação está sendo levada de uma parte do corpo para outra. Fator importante para transferências de proteínas e RNAs (mRNA e microRNA) entre as células, como base de vários processos fisiológicos e patológicos. As Evs incluem três subgrupos: exossomos, microvesículas e corpos apoptóticos (os quais são fragmentos dos núcleos das mutações cancerosas) e proteínas e materiais genéticos (CHENG; NONAKA; WONG, 2019). 
A Interleucina (IL) 4 está envolvida na invasão e metástase tumoral (AZIZ et al., 2015). A IL-6 e 8 estão envolvidas no processo de desenvolvimento de lesões orais pré- malignas, demonstrando a existência de maior expressão na saliva dos indivíduos diagnosticados com leucoplasia, ou seja, detecção precoce de lesões pré-malignas (SADAKSHARAM, 2015). Lembrando que a presença de úlceras aftosas e doença periodontal podem influenciar de forma negativa (AZIZ et al., 2015; SAHIBZADA et al., 2017).

O gene CD 44 codifica diferentes proteínas isofórmicas que estão relacionadas ao processo de malignização. No entanto, estes variam de acordo com o tipo de câncer. No caso do câncer da cavidade oral, estão associados a neoplasias malignas (BASAKRAN, 2015).

Vários estudos já demonstraram a utilidade da saliva para o diagnóstico e condições reais da saúde do indivíduo, como pacintes portatores de Diabetes mellitus, Vírus da Imunodeficiência Humana (HIV), doença cardíaca, doenças auto-imunes e uso de tabaco. Assim, muitos investigadores vêm tentando usar a saliva para avaliar a saúde (CHENG; NONAKA; WONG, 2019).

Os estudos proteômicos da saliva revelaram que 20 a $30 \%$ do proteoma salivar espelha o proteoma plasmático, indicando que uma porção substancial de constituintes salivares são derivados do sangue. Assim, a sobreposição significativa entre a saliva e o sangue devido a suas interações fisiológicas indicam uma aproximação alternativa potencial para diagnosticar doenças sistêmicas (KHAN et at., 2018).

\section{CONCLUSÕES}

A biópsia líquida surgiu nas últimas décadas com a proposta de ser não invasiva para uso na medicina personalizada e preventiva. Assim, a detecção e análise dos biomarcadores representam uma oportunidade promissora para 0 diagnóstico precoce do câncer, para investigação molecular, monitoramento da resposta ao tratamento e detecção de doença residual.

Em comparação com outros tipos de câncer, o oral tem como vantagens sua localização. Todavia, o impacto das biópsias líquidas no exame clínico ainda é restrito, exigindo mais pesquisas para uma implementação efetiva tanto dos profissionais, como em equipamentos para os exames, o que facilitaria para os médicos e cirurgiões dentistas, possibilitando uma condição melhor de tratamento. 
Mais informações e conhecimento da biologia oral e da origem dos biomarcadores circulantes específicos são fundamentais para 0 desenvolvimento de terapias eficazes para o manejo do câncer bucal. A biópsia líquida ainda está no começo, então os esforços da pesquisa devem ser direcionados para realizar estudos prospectivos multicêntricos que investiguem o papel dos biomarcadores salivares para melhor prognóstico destes pacientes.

\section{REFERÊNCIAS BIBLIOGRÁFICAS}

1. AMERICAN CANCER SOCIETY. Global cancer facts \& figures. Disponivel em: <https://www.cancer.org/research/cancer-factsstatistics/global.html>. Acesso em: 13 abr. 2021.

2. AZIZ, S.; AHMED, S.S.; ALI, A.; KHAN, F.A.; ZULFIQAR, G.; IQBAL, J. et al. Salivary immunosuppressive cytokines IL-10 and IL-13 are significantly elevated in oral squamous cell carcinoma patients. Cancer Investigation, v.33, n.7, p.318-328, 2015.

3. BANO, S.; DAVID, M.P.; INDIRA, A.P. Salivary biomarkers for oral squamous cell carcinoma: an overview. indian association of oral \& maxillo-facial pathologists. IJSS Case Reports and Reviews, v.1, n.8, p.39-45, 2015.

4. BASAKRAN, N.S. CD44 as a potential diagnostic tumor marker. Saudi Medical Journal, v.36, n.3, p.273-279, 2015.

5. BELLAIRS, J.A.; HASINA, R.; AGRAWAL, N. Tumor DNA: an emerging biomarkers in head and neck cancer. Cancer Mestasis Reviews, v.36, n.3, p.515-523, 2017.

6. CARNIELLI, C.M.; MACEDO, C.C.S.; DE ROSSI, T.; GRANATO, D.C.; RIVERA, C.; DOMINGUES, R.R. et al. Combining discovery and targeted proteomics reveals a prognostic signature in oral cancer. Nature Communications, n.9, ID:3598, 2018.

7. CARVALHO, J.; OLIVEIRA, C. Extracellular vesicles-powerful markers of cancer evolution. Frontiers in Immunology, v.5, article 685, p.1-2, 2015. 
8. CHENG, J.; NONAKA, T.; WONG, W.T. Salivary exosomes as nanocarriers for cancer biomarker delivery. Materials, v.12, n.4, p.654671, 2019.

9. CHENG, Y.S.L.; JORDAN, L.; GORUGANTULA, L.M.; SCHNEIDERMAN, E.; CHEN, H.S.; REES, T. Salivary Interleukin-6 and 8 in patients with oral cancer and patients with chronic oral inflammatory diseases. Journal of Periodontology, v.85, n.7, p.956-965, 2014.

10. CHI, A.C. Patologia epitelial. In: NEVILLE, B.W.; DAMM, D.D.; ALLEN, C.M.; BOUQUOT, J.E. Patologia oral e maxilofacial. 3a ed. Rio de Janeiro: Elsevier, 2009. Cap.10, p.363-454.

11.FERLAY, J.; COLOMBET, M.; SOERJOMATARAM, I.; MATHERS, C.; PARKIN, D.M.; PINEROS, M. et al. Estimating the global câncer incidence and mortality in 2018: GLOBOCAN sources and methods. International Journal of Cancer, v.144, n.8, p.1941-1953, 2019.

12. GUPTA, P. Role of salivar biomarkers for early detection of oral squamous cell carcinoma. International Journal of Avanced \& Integrated Medical Sciences, v.2, n.3, p.155-160, 2017.

13. INCA - Instituto Nacional de Câncer. Estimativa 2018: incidência de câncer no Brasil. Rio de Janeiro: INCA, 2017.

14.KACZOR-URBANOWICZ, K.E.; CARRERAS-PRESAS, C.M.; ARO, K.; TU, M.; GARCIA-GODOY, F.; WONG, D.T. Saliva diagnostics - Current views and directions. Experimental Biology and Medicine, v.242, n.5, p.459-472, 2017.

15.KHAN, M.R.S.; SIDDIKA, F.; XU, S.; LIU, X.L.; SHUANG, M.; LIANG, H.F. Diagnosing oral squamous cell carcinoma using salivary biomarkers. Bangabandhu Sheikh Mujib Medical University, n.11, p.1-10, 2018.

16.KHURSHID, Z.; ZOHAIB, S.; NAJEEB, S.; ZAFAR, M.S.; SLOWEY, P.D.; ALMAS, K. Human saliva collection devices for proteomics: an update. International Journal of Molecular Sciences, v.17, n.6, p.846, 2016. 
17.LOUSADA-FERNANDEZ, F.; RAPADO-GONZALEZ, O.; LOPEZCEDRUN, J.L.; LOPEZ-LOPEZ, R.; MUINELO-ROMAY, L.; SUAREZCUNQUEIRO, M.M. Liquid biopsy in oral cancer. International Journal of Molecular Sciences, v.19, n.6, p.1704-1718, 2018.

18.MORO, J.S.; MARONEZE, M.C.; ARDENGHI, T.M.; BARIN, L.M.; DANESI, C.C. Câncer de boca e orofaringe: epidemiologia e análise da sobrevida. Einstein (São Paulo), v.16, n.2, eAO4248, 2018.

19. OMS - Organização Mundial de Saúde. Iniquidades sociais e dos cuidados de saúde na incidência e mortalidade por câncer. Disponível em: <https://doi.org/10,32635/2176- 9745.RBC.2018v64n4.211>. Acessado em: 13 abr. 2021.

20.RADHIKA, T.; NADEEM, J.; NITHYA, S.; MUTHUMEENAKSHI, R.M. Salivary biomarkers in oral squamouss cell carcinoma - an insight. Journal of Oral Biology and Craniofacial Research, v.6, (Suppl), p.51-54, 2016.

21. SADAKSHARAM, J. Advances in diagnosis of oral potentially malignant disorders: a review. Journal of Indian Academy of Oral Medicine and Radiology, n.27, p.169-170, 2015.

22. SAHIBZADA, H.A.; KHURSHID, Z.; KHAN, R.S.; NASEEM, M.; SIDDIQUE, K.M.; MALI, M. et al. Salivary IL-8, IL-6 and TNF-a as potential diagnostic biomarkers for oral câncer, Diagnostics (Basel), v.7, n.2, pii: E21, 2017.

23. SAXENA, S.; SANKHLA, B.; SUNDARAGIRI, K.; BHARGAVA, A. A review of salivary biomarker: a tool for early oral cancer diagnosis. Advanced Biomedical Research, v.6, n.1, p.90, 2017.

24.SHAH, F.D.; BEGUM, R.; VAJARIA, B.N.; PATEL, K.R.; PATEL, J.B.; SHUKLA, S.N.; et al. A review on salivary genomics and proteomics biomarkers in oral cancer. Indian Journal of Clinical Biochemistry, v.26, n.4, p.326-334, 2011.

25. SINEVICI, N; O'SULLIVAN, J. Oral cancer: deregulated molecular events and their use as biomarkers. Oral Oncology, n.61, p.12-18, 2016. 
26. WANG, K.; LU, W.; TU, Q.; GE, Y.; HE, J.; ZHOU, Y. et al. Preliminary analysis of salivary microbiome and their potential roles in oral lichen planus. Scientific Reports, n.6, Id:22943, 2016.

27.ZORZETTO, R. No sangue, mais pistas sobre o câncer. Pesquisa Fapesp, n.253, p.51-55, 2017. 